\title{
Surface micro-profilometry for the assessment of the effects of traditional and innovative cleaning treatments of silver
}

\author{
Claudia Daffara ${ }^{1 \star}$, Nicola Gaburro ${ }^{1}$, Giacomo Marchioro ${ }^{1}$, Alessandro Romeo ${ }^{1}$, \\ Giulia Basilissi ${ }^{2}$, Andrea Cagnini ${ }^{2}$, Monica Galeotti ${ }^{2}$ \\ 1 Department of Computer Science, University of Verona, Strada Le Grazie 15-37134 Verona, Italy \\ 2 Opificio delle Pietre Dure, Viale F. Strozzi 1-50129 Firenze Italy \\ * Corresponding author: claudia.daffara@univr.it
}

\begin{abstract}
In the complex procedure for the conservation of a work of art, the effective monitoring of the surface treatment is an important step in order to establish a conservation protocol. The evaluation of the effects of the cleaning requires the inspection of the outermost layer with complementary diagnostic techniques to obtain information on both materials and surface morphology.

In this interdisciplinary work, a custom optical micro-profilometry device based on laser conoscopic holography was used for testing the performance of different mechanical procedures for the cleaning of silver alloy objects.

Tarnishing is the main alteration phenomenon for silver alloy artworks. Even if such modification does not affect the long-term preservation of the objects, it heavily influences aesthetical features, making the removal of tarnishing one of the most important conservation treatments of the case. The mechanical cleaning procedure based on abrasive powders suspended in a liquid matrix is of widespread use. Recently, a novel dry cleaning procedure based on the use of erasers was proposed as alternative method and tested for both the efficacy of tarnishing removal and effects on the surface appearance using optical microscopy and scanning electron microscopy. However, the employed laboratory techniques cannot provide an objective measure of the roughness at micrometric scale over the sample.

In this work, the effects on the surface structure of traditional and innovative silver treatments was investigated with multiscale profilometry, using scanning conoscopic holography and atomic-force microscopy. 3D surface metrology was carried out using standard areal amplitude parameters and a multiscale approach.

Preliminary results are presented, addressing two aspects: 1) the validation of laser optical micro-profilometry for the diagnostics of silver objects, which are challenging due to the highly specular surface; 2) the validation of the dry-cleaning treatment based on rubbers, by adding to the previous study the complimentary analysis of the micro-surface. The results obtained on silver mockups confirm that the proposed dry cleaning methods produce surface variations less or similar to traditional methods. The optical micro-profilometry results are of particular significance since this technique is portable with wide field capability, thus allowing an in-situ use and a real monitoring of actual artwork surfaces.
\end{abstract}

Keywords: optical micro-profilometry, conoscopic holography, silver cleaning, surface analysis, artworks 


\section{Introduction and background}

The degradation of silver is an important topic in conservation science. Many ancient artefacts were made of silver but this precious metal is more sensitive to degradation compared to other noble metals such as gold and for this reason requires special care to preserve the original surface. Silver degradation has been studied in different environments [1] and extensive studies have been carried out regarding the embrittlement of buried silver objects $[2,3]$. One of the main degradation processes affecting silver artefacts is the corrosion due to atmosphere gases [4]. This is nowadays one of the most critical issue for objects conserved in collections.

Tarnishing is the main alteration phenomenon for silver alloy artworks due to the interaction of silver with sulphur-containing compounds in the environment. Such modification heavily influences aesthetical features, making the removal of tarnishing one of the most important conservation treatments of the case. Different cleaning procedures are proposed, based on mechanical, electrochemical, chemical and physical methods [5]. The mechanical procedure utilizing an abrasive powder suspended in a liquid matrix is of widespread use due to its simplicity and effectiveness. Despite these advantages, issues are raised concerning the cleaning of objects in which more materials coexist near to or above silver (enamels, other metals, and organics). In these cases, the use of water- or solvent-based means is often not advisable. To address this point, the Opificio delle Pietre Dure has recently introduced a dry cleaning process based on the use of erasers as alternative to traditional mechanical methods [6]. Several rubber-based methods have been tested regarding the efficacy of tarnishing removal and the effects on the surface appearance. Preliminary tests have proven that such innovative procedure effectively removes tarnishing and has been recently used in the final phase of the cleaning of the silver "agemina" letters in the North Door by Lorenzo Ghiberti of the Baptistery of Florence in Italy.

The evaluation of the effects of the cleaning requires the outermost layer of the artwork to be inspected with complementary diagnostic techniques to gain information on both materials and surface morphology. Different studies regarding the choice of the best cleaning methodology have been carried out using routine benchtop techniques on tarnished silvers mock-ups $[7,8]$ to control mass loss and surface variation after the cleaning process. However, most of these techniques can analyse only a small area of the sample and cannot be brought in situ.

In this work, we used a newly developed optical micro-profilometer based on 3D scanning conoscopic holography that can be brought in the place where artefacts are conserved for an in-situ monitoring [9]. Laser conoscopic holography is a non-contact technique that has been developed in the last decades [10]. It provides distance measurements in non-invasive way in a versatile working range allowing the acquisition of artwork surfaces at different scales with micrometric resolution. This technique has proven its potential on different applications, e.g. archaeology [11], laser cleaning [12], painting conservation [13]. Silver material, which is highly specular, is particularly challenging.

Here, laser conoscopic holography was applied to monitor the surface in different mechanical procedures for the cleaning of silver alloy objects, including traditional 
treatments and the innovative dry-cleaning methods based on rubbers.

This interdisciplinary work addresses two aspects, one related to the instrumental tool, the other related to the specific conservation application. The aims are:

- to validate laser optical micro-profilometry as an effective diagnostic tool for the analysis of silver objects, which are challenging due to the highly specular surface;

- to further validate a new cleaning treatment for silver, adding to the previously carried out study [6] the complimentary analysis of the micro-surface.

\section{Materials and methods}

\subsection{Silver cleaning samples}

A set of silver alloy mock-ups was prepared reproducing different mechanical cleaning treatments. The original untreated silver surface was finished using abrasive paper of different grain sizes, with the polishing carried out manually along a fixed direction.
Two samples were treated with traditional mechanical methods (calcium carbonate and sodium hydrogen carbonate suspended in water) and five samples were treated with the innovative dry cleaning method based on erasers. Five rubbers were employed with different characteristics and abrasive power. The cleaning treatments were performed by ten crisscross applications each. After scrubbing, the surface was washed with acetone, where the erasers were used, and with water where abrasive powders were used. The Table 1 reports the silver cleanings samples specifications. The rubber-based cleaning tools contain abrasive particles of different size and distributions.

The efficacy of removal of silver sulphide was studied using optical microscopy and SEM in a previous work [6] while this study is aimed at investigating the effects on the surface trough a quantitative roughness analysis. In fact, though laboratory microscopy techniques, which are being used for the inspection of surface morphology alteration, are proven to detect the single defects locally,

Table 1. Silver cleaning treatments

\begin{tabular}{cll}
\hline Sample & Cleaning treatment & Abrasive medium \\
\hline A & $\begin{array}{l}\text { Sodium hydrogen carbonate suspended } \\
\text { in water }\end{array}$ & Sodium bicarbonate, grain size $300-500 \mu \mathrm{m}$ \\
\hline B & Calcium carbonate suspended in water & Calcium carbonate, grain size about $100 \mu \mathrm{m}$ \\
\hline C & Rubber Staedtler refill & $\begin{array}{l}\text { Calcium carbonate, grain size } 1-30 \mu \mathrm{m}, \\
\text { organic matrix medium with S and Cl }\end{array}$ \\
\hline D & Rubber Cretacolor Monolith & $\begin{array}{l}\text { Calcium carbonate, grain size } 1-50 \mu \mathrm{m} \text { with } \\
\text { higher density and same composition of } \\
\text { sample C }\end{array}$ \\
\hline E & Rubber pencil Perfection Faber-Castel & $\begin{array}{l}\text { Calcium carbonate, grain size } 1-35 \mu \mathrm{m} \text { with } \\
\text { homogeneous density. No S and Cl }\end{array}$ \\
\hline F & Dental wheel rubber Wilpas & $\begin{array}{l}\text { Silicon rubber. Zirconium of grain size 1-10 } \\
\mu \mathrm{m} \text { with homogeneous distribution }\end{array}$ \\
\hline G & Dental pointed rubber Identoflex & $\begin{array}{l}\text { Silicon rubber. Aluminum oxide of grain size } \\
1-10 \mu \mathrm{m} \text { with homogeneous distribution }\end{array}$ \\
\hline
\end{tabular}


they cannot provide an objective measure of the surface features at micrometric scale over the sample.

In this experiment, the cleaning treatments were applied directly to the silver surface without inducing artificial tarnishing in order to focus on the effect of the mechanical abrasive process on the surface.

\subsection{Surface measurements}

The measured roughness is not an intrinsic property but is dependent on the bandwidth of the measurement [14]. The distribution of size and shape of the asperities in the digitized surface is limited by the sampling step, which determines the shortest spatial structure, and by the sampling length, which determines the longest one. The information collected should refer to the range of surface features determined by the specific application.

For acquiring the surface at micrometric level we have used a custom optical micro-profilometer that has been implemented in the framework of the Scan4Reco European project [15]. The system is based on a laser conoscopic holography probe mounted on two linear micrometric stages $\left(30 \times 30 \mathrm{~cm}^{2}\right.$ scan size). While the motion system scans the sample, the probe measures the distance to the surface, thus collecting a sequence of heights that allows the reconstruction of the surface map. Measurement range, lateral resolution, and standoff distance vary with the probe-lens coupling and can be tailored to the scale of the object (from sub-millimetres to centimetres). For the silver cleaning samples a ConoPoint-3R sensor (by Optimet) coupled to a $25 \mathrm{~mm} \mathrm{~N}$ lens was used, with a measurement range of $1 \mathrm{~mm}$ and a standoff distance of $16 \mathrm{~mm}$. The probe has a lateral resolution of 5 micron determined by the laser spot and a repeatability of 0.06 micron. In this study, the surface data has been acquired using a stage step of 5 micron. The entire size of the samples has been acquired.

The samples have been measured also with Atomic Force Microscopy (AFM) to provide supplementary surface data at sub-micron scale. AFM allows the measurement of fine details of the surface but in a small scan size. AFM measurements have been carried out with a NT-MDT -SMENA A with a goldcoated silicon tip from the same company in semi-contact mode. Five scans have been taken for each sample with a scan length of 60 micron (256 sampled points).

\subsection{Surface data analysis}

The surface geometrical structure has a multi-scale nature being a superimposition of a large number of scales of roughness. The common approach in surface metrology [16] is to describe a surface profile in terms of components of different bandwidth along the scan length: the roughness, i.e. irregularities at smaller wavelengths, the waviness, i.e. more widely spaced variations, and the shape (Fig. 1). Such features are usually ascribed to deviations from an "ideal" surface; for instance, the roughness can be put in relation with the manufacturing process inherent to the material, while the waviness with the quality of the process, e.g. presence of vibration. The cut-off value, i.e. the length of the scale at which the roughness is isolated, should be determined by the application by putting in relation these scale-limited features to the process in which are generated. However, in the cultural heritage field the artistic materials are not created in controlled processes such as in engineering and the artwork is being subjected to events that make 

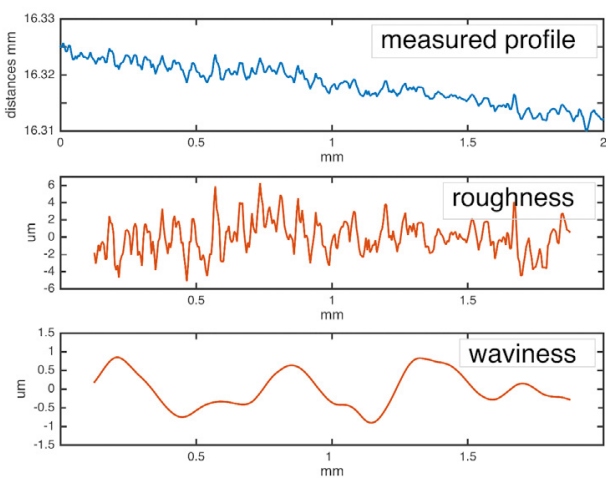

Fig. 1. Example of a profile measured by the optical profilometer and the roughness and waviness components for a cut-off of 250 micron ( $2 \mathrm{~mm}$ detail from sample A).

its surface history complex (and unknown) including materials' degradation and manual treatments.

From the scanning profilometry measurements we obtain a 2D map of heights, hence, a $3 \mathrm{D}$ points cloud that models the real surface in the bandwidth of the measurement. This digital representation is processed for extracting the parameters that describe the surface quality according to the interested features. Inspection of surface structure includes the detection of local defects and global roughness. Here, we are mainly concerned with the roughness aspect and even if the optical micro-profilometry is shown capable to detect fine local defects in silver, e.g. scratches, strokes, they are taken into account only in relation to the roughness amplitude contribution without their localization.

Different kinds of metrological parameters are available for roughness evaluation [17] and the ability of discriminating the topography can depend on this choice. In this study, surface evaluation is performed using areal parameters recommended by the standards [18], in terms of:
1) global features based on the average behaviour of the surface amplitude, by calculating the roughness parameter $S q$ (root mean square of the height distribution);

2) isolated events (i.e. defects), by calculating the extreme values parameters: $S p$ (maximum height of peaks), $S v$ (maximum height of valleys), and $S z$ (maximum height of the surface);

3) a multiscale approach, by inspecting the variation of the roughness features with the scale.

In order to characterize the surface topography, the first step was the removal of the form from the measured data. The analysis of the surface was then carried out with a multiscale approach as follows. As a first step, the roughness was analysed in a local representative region, and then the variation of the roughness distribution over the sample was evaluated. As a second step, the separation of the roughness and the waviness components was performed on the whole sample for different values of cut-off (Fig. 2). Roughness parameters were then calculated both on the total surface than on the scale-limited components. As a third step, the variation of the roughness parameters with the observation scale, i.e. the scan area, was inspected.

\section{Results}

\subsection{Micro-profilometry}

The surface texture generated by the different cleaning procedures is shown in Fig. 3 for comparison. For each sample it is reported the $3 \mathrm{D}$ plot of a central ROI after form removal and mean subtraction. The structure of the surface presents peaks, deep valleys, and plateaus due to the different cleaning treatment, with the amplitude density func- 


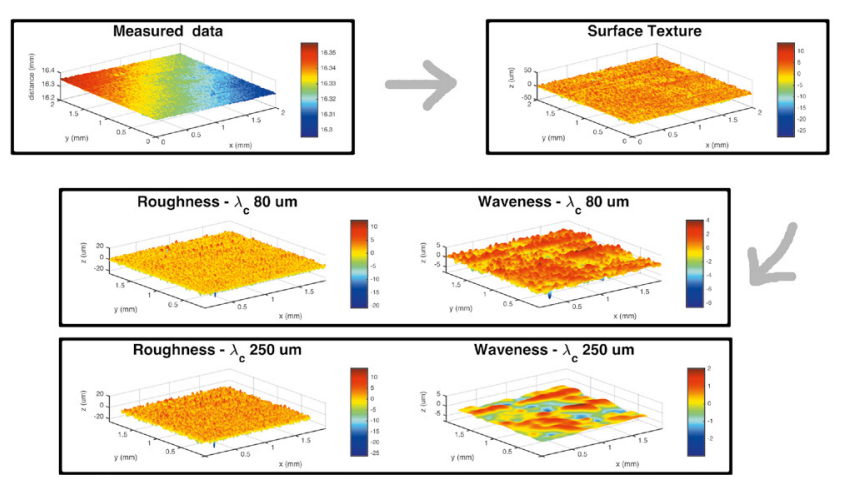

Fig. 2. Scheme of surface processing $\left(2 \times 2 \mathrm{~mm}^{2}\right.$ detail from sample A): from measured surface data to surface texture after form removal with polynomial best fit; extraction of roughness and waviness by Gaussian filtering with different cut-off values (example with 80 and 250 micron).
A

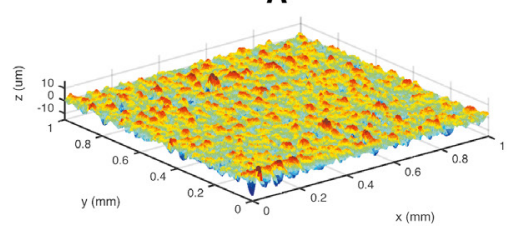

C

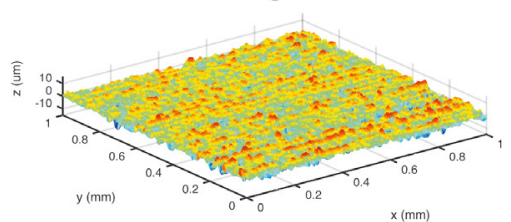

E

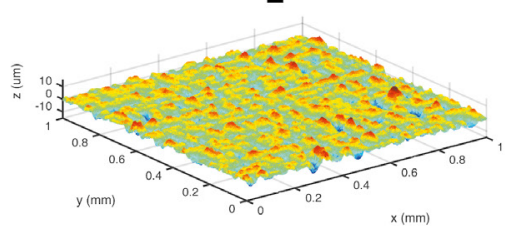

G

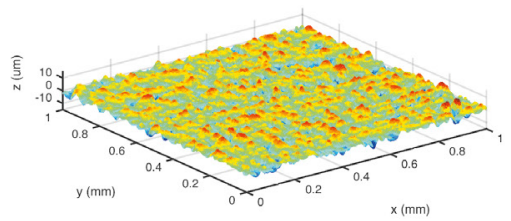

B
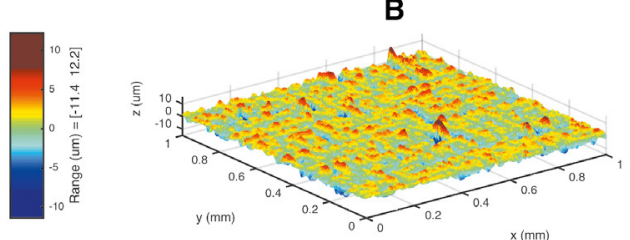

D
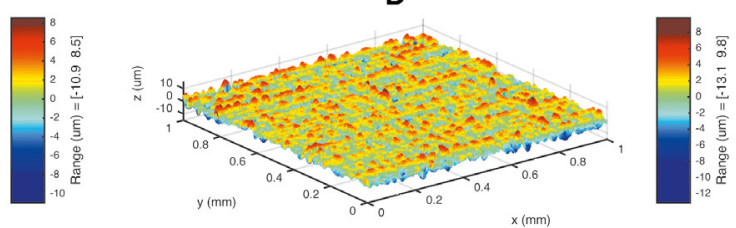

F

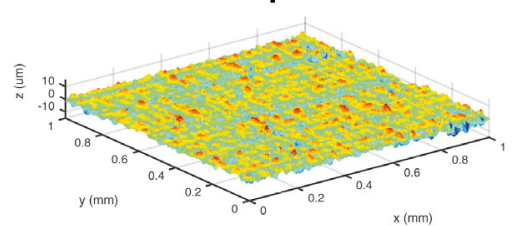

Non treated

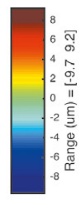

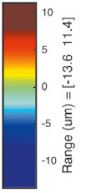

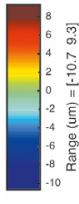

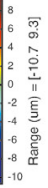

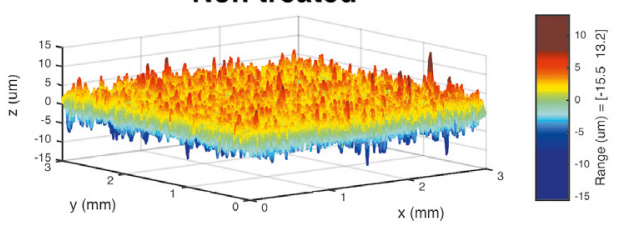

Fig. 3. 3D mesh plot of the micro-surface texture of each cleaning sample $\left(1 \times 1 \mathrm{~mm}^{2} \mathrm{ROI}\right)$ and of the nontreated silver sample $\left(3 \times 3 \mathrm{~mm}^{2} \mathrm{ROI}\right)$. 
tion (ADF) in a local ROI of nearly Gaussian characteristic (Fig. 4).

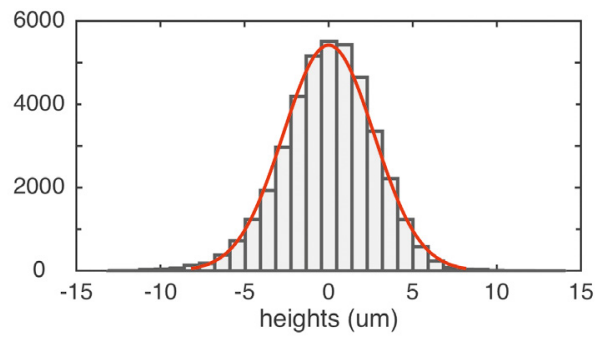

Fig. 4. Example of amplitude distribution of a local ROI $\left(1 \times 1 \mathrm{~mm}^{2}\right)$ in sample A with the fitted normal distribution.

In the first phase of the analysis, the $S q$ roughness was evaluated in a central ROI of $2 \times 2 \mathrm{~mm}^{2}$, after removing the outliers and averaging three different measurements taken with the sample rotated in different positions. In general, the roughness generated by the erasers is similar (order of micron) to the one generated by the abrasive powders showing that the dry cleaning process is compatible with the traditional methods (Fig. 5). The roughness is higher for the sample cleaned with the sodium bicarbonate $(\mathrm{A})$ and lower for the two samples treated with the dental tools ( $F$ and $G$ ) containing zirconium grains;

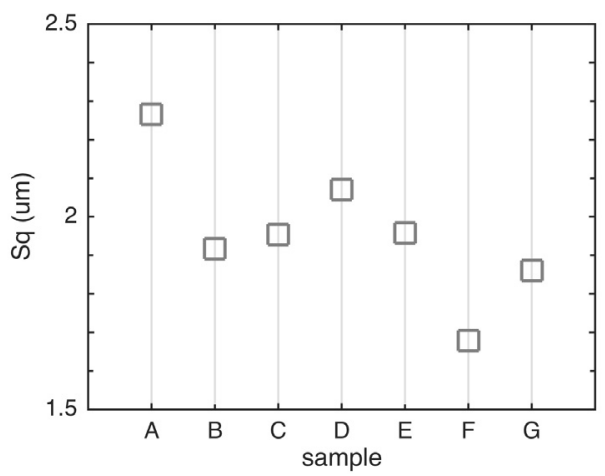

Fig. 5. Roughness $S q$ of the cleaning samples calculated in a representative ROI of $2 \times 2 \mathrm{~mm}^{2}$. the rubber tools containing grains of calcium carbonate $(\mathrm{C}, \mathrm{D}$, and $\mathrm{E})$ generate a roughness similar to the one generated by the calcium carbonate powder (B). The differences in the $S q$ roughness are compatible with the sizes of the abrasive grains observed with SEM and reported in Table 1. In an abrasion process there is expected a decrease of the amplitude roughness with the decrease of the abrasive grain size [19].

The roughness amplitude in the above-evaluated local ROIs is found to follow a nearly Gaussian distribution, as expected in relation to the underlying abrasion process. Abrasion is a very complex mechanism that involves phenomena related to the microstructural properties of the abrasive and the abraded materials and to the kind of process applied. The final roughness is influenced by a large number of factors, above all the hardness, the morphology and the size of the abrasive particles [20]. In principle, when an invasive mechanical cleaning is applied homogeneously to the surface, the overall roughness is lowered and if the process is applied for enough time the final roughness tends to a stationary value that is characteristics of the cleaning method, which is determined by the size and distribution of the abrasive particles contained in the cleaning tool. However, the cleaning process may not be homogeneous, some area may be cleaned for longer time or with higher pressure compared to others and this can lead to an inhomogeneous surface with a higher roughness (Fig. 6).

If the sampling area is too small compared to the inhomogeneity of the surface, these differences cannot be measured properly. Inhomogeneity of the surface roughness over the sample can be inspected by looking at the distribution of the averaged roughness obtained by dividing the sample in sub-re- 
Original surface $\mathrm{Rq}=0.142$

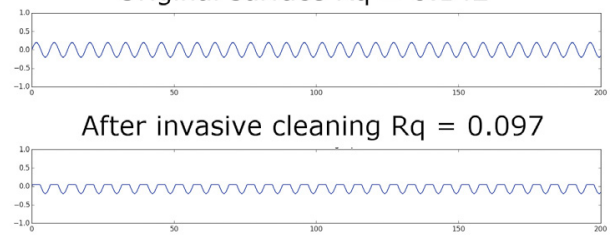

After inhomogeneous cleaning $\mathrm{Rq}=0.108$

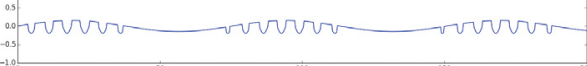

Fig. 6. Effect of inhomogeneous cleaning on the roughness. In the example, obtained using synthetic data in arbitrary units, the final roughness is given by the sum of contributes from the peak abrasion and from the waviness due to the inhomogeneous process.

gions of $50 \times 50$-sampled points (squares of 250 micron side). Here, the dry cleanings are shown to generate a homogeneous roughness, with the coefficient of variation (standard deviation normalized by the mean) of the roughness distribution compatible with the traditional methods (Fig. 7). As example, the averaged roughness map obtained for

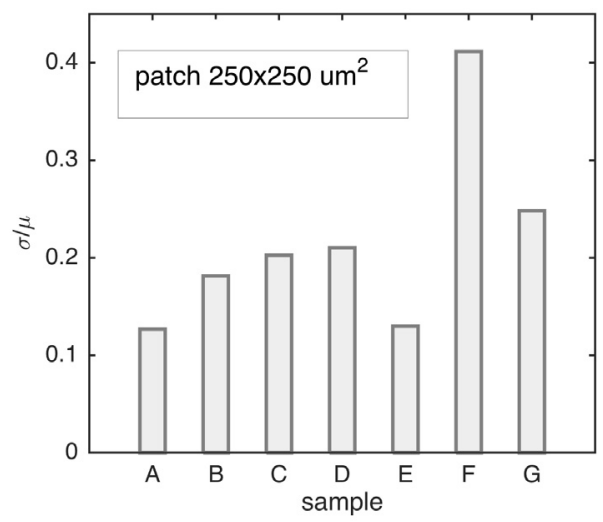

Fig. 7. Relative standard deviation (std dev/mean) of the averaged roughness distribution after dividing the sample in sub-regions of $250 \times 250 \mu \mathrm{m}^{2}$ for different treatment methods (Table 1 ). The rubber-based cleanings generate a surface roughness with a dispersion compatible to the traditional powders method $(A, B)$, with exclusion of the dental wheel (F). the samples treated with traditional and dry cleaning is shown in Fig. 8.

In the second phase of the analysis, the roughness and the waviness have been separated over the whole sample using Gaussian filtering for the following sequence of cut-off: $50,100,200,250,500,800$, and 1000 microns. The multiscale analysis shows a similar roughness behaviour for the dry cleaning treatments and the traditional methods. In addition, the roughness calculated in the scale-limited components points out three
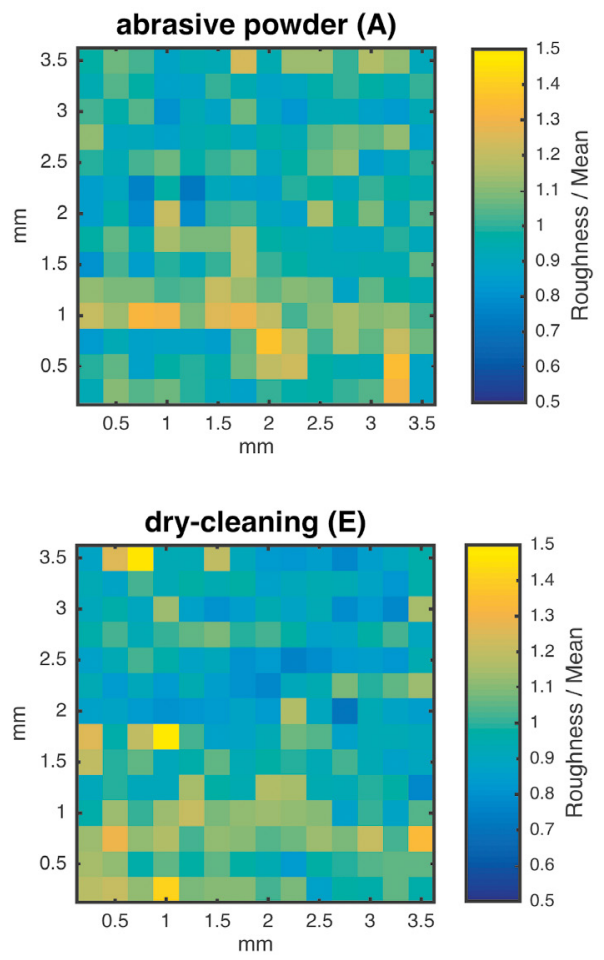

Fig. 8. Map of the roughness averaged in cells of 250 $\times 250 \mu \mathrm{m}^{2}$ and normalized by the mean of the cells for comparison: sodium bicarbonate abrasive powder (A) and dry cleaning with the rubber "Perfection" (E). The interval $0.5-1.5$ is plotted, with all the values outside assigned to the extremes of the colour map, to separate the effects of isolated peaks and valley and inspect the homogeneity. 
distinct groups (Fig. 9 and Fig. 10): the samples treated with abrasive powders (A and $B)$, which have the higher roughness at the various cut-off, the samples treated with the calcium carbonate rubbers $(\mathrm{C}, \mathrm{D}$, and $\mathrm{E})$

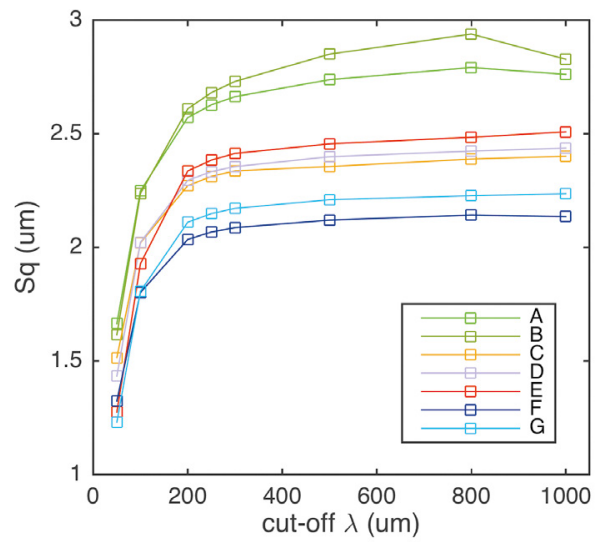

Fig. 9. Roughness $S q$ of the samples calculated for different scale-limited components using a Gaussian filter and a sequence of cut-off in the range from 50 $\mu \mathrm{m}$ to $1 \mathrm{~mm}$.

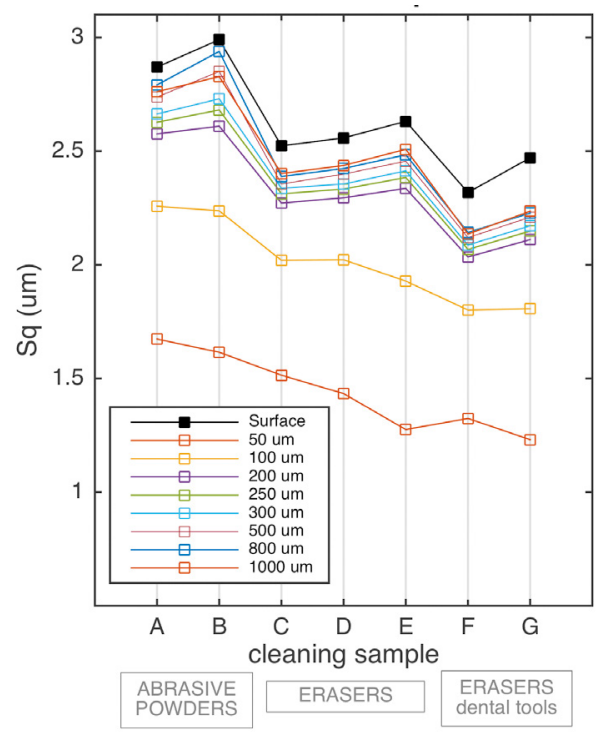

Fig. 10. Roughness $S q$ of the total surface and scale-limited components plotted versus the method of treatment (Table 1). with intermediate values of roughness, and the dental tools based on zirconium grains ( $F$ and $G$ ), which have the lower level of roughness. At the lower cut-off the contribute should be put in relation with the abrasive medium while at the longer scale with the waviness contribute induced by the kind of process over the sample.

The examined treatments have the same nature of a statistical abrasive process but the mechanical removal of the material at microscopic level is different. In fact, in the cleaning with the abrasive powder the abrasive particles are free, suspended in a liquid, while in the dry cleaning with the rubbers the abrasive grains are bonded to the tool. The observed differences in the roughness in the scale-limited components therefore depend on both microscopic and macroscopic factors, from the kind of abrasive mechanism, the kind of abrasive particles, to the manual procedure applied by the restorer (pressure, path in which the tool is moved, etc.).

Due to the multiscale nature of the surface the roughness may increase with the size of the evaluation area, but roughness variations may be ascribed also to the surface treatment that accumulates defects resulting in isolated peaks or valleys. Independent scratches or marks with low periodicity increase the global roughness. In order to consider this aspect, in the third phase of the analysis, the main roughness amplitude parameters were evaluated as function of the scan size using a multiscale approach [21]. To compute the multi-scale roughness it has been implemented an algorithm as follows. The surface is subdivided in square sub-regions of specific side; for each patch the extreme roughness amplitudes $S p$ (representing the peaks), $S v$ (representing the valley), $S z$ (representing the maximum amplitude range), together with the $S q$ roughness, are calculated; then 
the roughness parameters are averaged on the patches. This step is repeated varying the side of the patch. The roughness parameters are plotted versus the patch size, giving an insight of their variation with the observation scale (Fig. 11).

The plots show a similar behaviour for the dry cleaning methods and the traditional abrasive powders. The maximal and minimal roughness amplitudes increase with the scale due to the higher probability of encountering higher peaks or deeper valleys. Differences
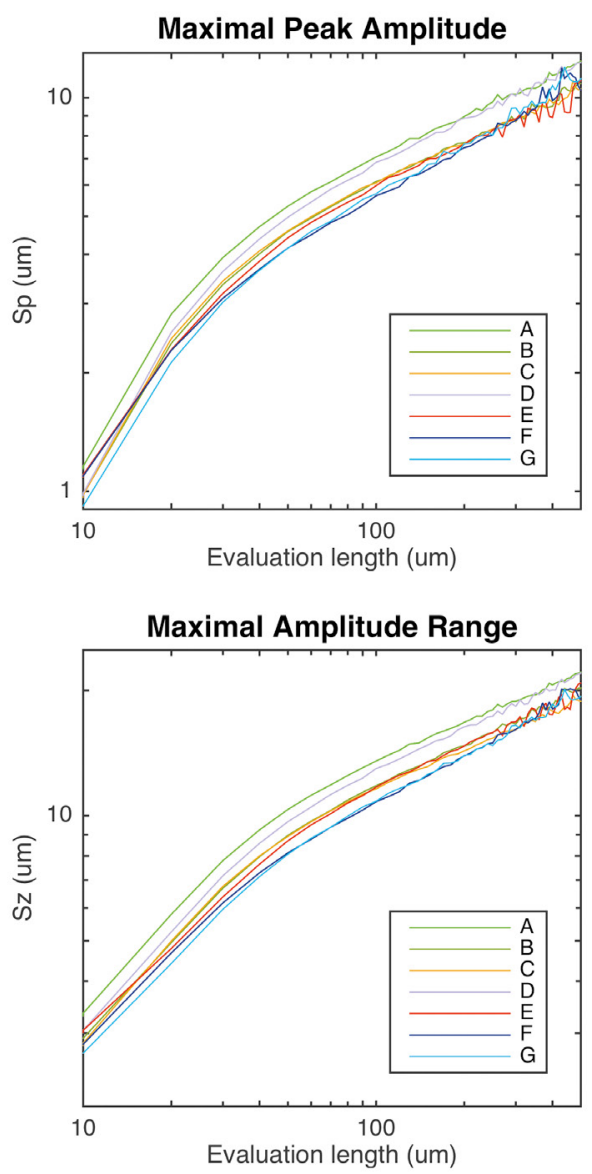

are related to the kind of abrasive particles and to the kind of process applied. The extreme amplitudes are higher for the sample treated with the sodium bicarbonate powder of larger grain size (400 micron), and lower for the samples treated with the rubbers containing the smaller zirconium grains (10 micron). Among the rubbers, the "Cretacolor Monolith" (D) exhibits higher values of both maximal and minimal roughness. The other dry cleaning methods based on rubber containing calcium carbonate show similar
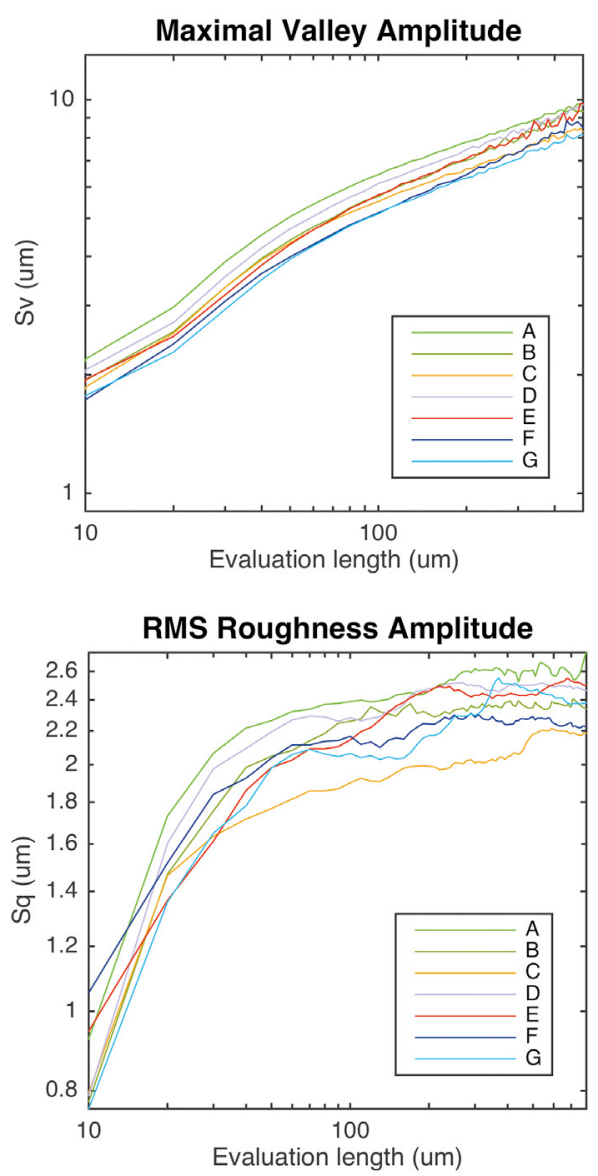

Fig. 11. Variation of the roughness parameters with the scan size (log-log plot): extreme amplitudes of peaks $S p$ (top-left), of valleys $S v$ (top-right), maximal surface heights $S z$ (bottom-left), RMS roughness $S q$ (bottom-right). 
values than the traditional powder method. Differently from the extreme amplitude roughness parameters, the $S q$ was found to tend to a constant value with the scale, meaning that there is a long-wavelength behaviour in relation to the underlying process.

\subsection{AFM analysis}

AFM measurements provided the supplementary evaluation of the sub-micron roughness locally (scan size $60 \times 60 \mu \mathrm{m}^{2}$ ). AFM surface map reveals the anisotropic surface structure with a predominant linear pattern in both the treated and non-treated samples (Fig. 12). This lay is due to the fact that the original surface of the silver sample has been
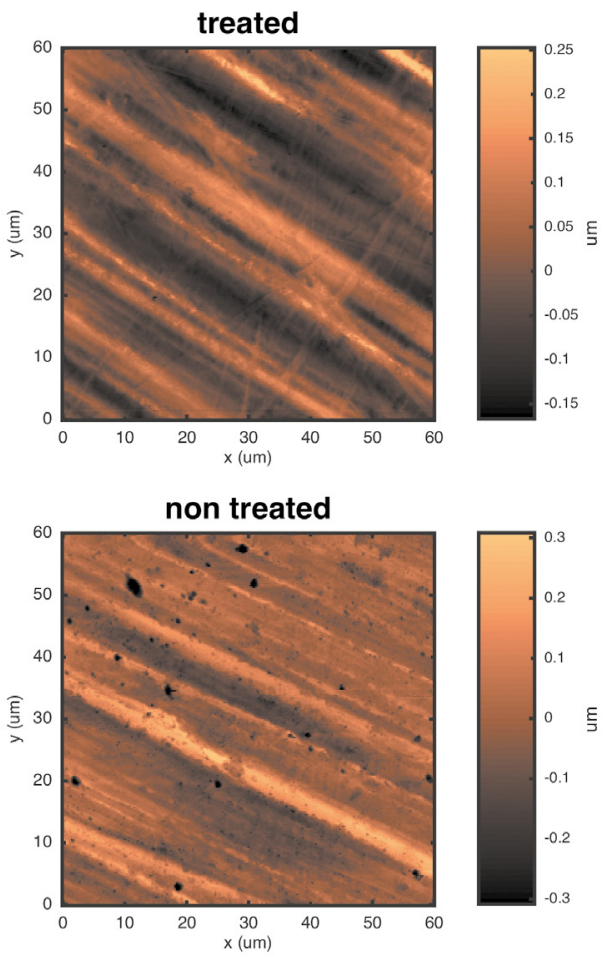

Fig. 12. AFM surface map (256pts, $60 \times 60 \mu \mathrm{m}^{2}$ scan size): treated (rubber) and non-treated silver sample. finished using sand paper along a single direction, thus engraving a regular grating in the surface. In the AFM surface map we can see the scratches due to the manufacturing process itself and the smaller scratches due to the cleaning process.

The roughness was evaluated by averaging $S q$ from five measurements taken for each samples. The comparison is reported in Fig. 13; here, the high values of uncertainty given by the error bars are due to the intrinsic variability of the roughness across the sample. Roughness is normally higher

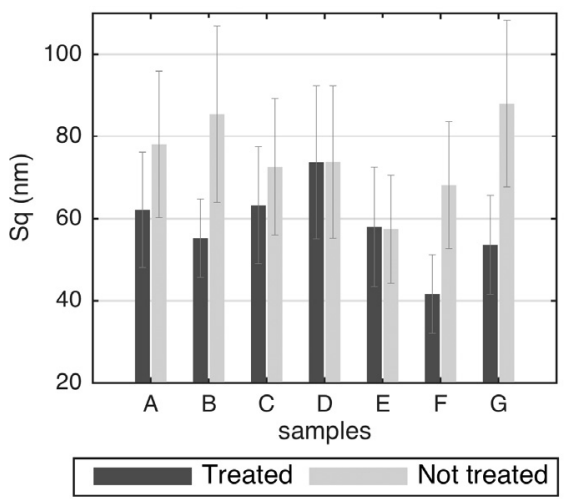

Fig. 13. AFM roughness of treated and non-treated silver sample for the different cleaning procedures. Roughness was obtained by averaging $S q$ from five different AFM scans for each sample; bar error is the standard deviation of the mean

for the reference sample and lower for the cleaned sample. Among the dry cleanings, the rubber-based treatments $\mathrm{D}$ and $\mathrm{E}$ exhibit the lower difference between the reference roughness and the sample roughness, the dental tools $F$ and $G$ produce higher surface variations.

\subsection{Discussion}

Most of the techniques used for retrieving the micro-surface of an object allow exam- 
ining only a small portion of the sample. An advantage of the set-up used in this work is the capability to measure objects with sizes up to $30 \times 30 \mathrm{~cm}^{2}$, together with keeping micrometric resolution, thus allowing the evaluation of low-periodicity features as waviness and shape deformation beside the roughness. Measuring the waviness requires such high depth precision as roughness but large scan sizes in order to evaluate the widely spaced periodicity variations.

Measuring the surface with the scanning micro-profilometer can provide a more effective way to estimate the cleaning inhomogeneity of the sample. In this case, the standard deviation of the heights distribution can generally provide an insight of the homogeneity. A visual evaluation of the surface topography map can also help to identify patterns left by the cleaning tool (Fig. 14).

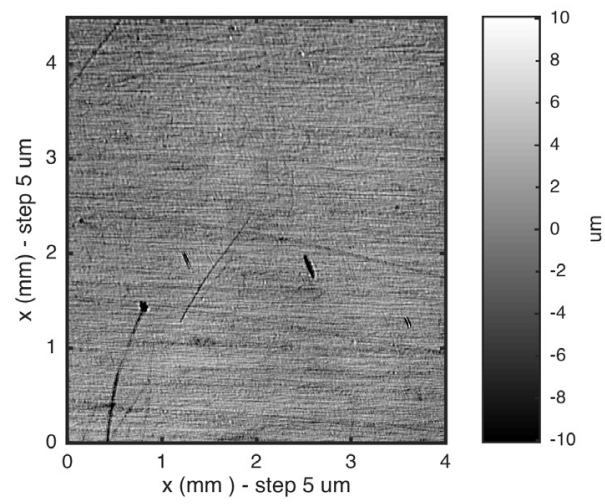

Fig. 14. Micro-profilometry surface map (2D intensity plot of the heights): detail showing the marks and scratches left by the cleaning procedure.

What is measured with the AFM technique is the roughness of the material, hence the invasiveness of the cleaning, while little information can be achieved regarding the homogeneity of the treatment. In this case, it is expected that a lower roughness ascribe a more invasive cleaning method.
Micro-profilometry and AFM allowed the evaluation of roughness features of different length scales depending on the bandwidth of the device. An (ideal) selection criterion should be to choose the cleaning method that best preserves the original roughness at the different scales.

\section{Conclusions}

The effectiveness of laser conoscopic holography in the analysis of the cleaning of silver, has been demonstrated, namely:

1. the technique is able to measure specular surfaces;

2. the bandwidth of the measurement yields to the representation of a relevant number of asperities, i.e. the distribution of shape and size of irregularities allows a reliable description of the main structures of the surface;

3 . the length scales of the measured roughness features (waviness and roughness) is relevant for the application, and some aspects of the surface topography are put in relation with the kind of material processing.

The optical micro-profilometer provides a more effective way to estimate the quality of the cleaning process in relation to the roughness inhomogeneity of the sample, including defects accumulation.

The multiscale approach allows a more accurate surface analysis. Anyway, understanding which is the relevant analysis scale is difficult. Given the multiscale nature of the roughness, as well as the multiscale capability of the optical profilometric probes, complementary measurement techniques with different bandwidths should be used for the characterization of the surface for a more comprehensive evaluation of the effective- 
ness of the cleaning method. To this respect, a further work is being planned to integrate optical micro-profilometry and AFM.

Concerning the aspect of surface conservation, the results obtained on a set of untarnished silver samples treated with both wet and dry cleaning methods point out that the rubber-based treatment produces a surface characterized by a roughness smaller or similar to traditional methods.

The optical scanning profilometry results are of particular significance since this technique allows an in-situ use for monitoring real artwork surfaces.

\section{Acknowledgements}

This work was supported by the Scan4Reco project funded by EU Horizon 2020 Framework Programme for Research and Innovation under Grant Agreement no 665091 .

\section{References}

[1] M.B. Mcneil, B.J. Little, "Corrosion Mechanisms for Copper and Silver Objects in Near-Surface Environments", Journal of the American Institute for Conservation, 31 (1992) 355-366, DOI: 10.2307/3179729.

[2] R.J.H. Wanhill, "Brittle Archaeological Silver: A Fracture Mechanisms and Mechanics Assessment", Archaeometry, 45 (2003) 625-636, DOI: 10.1046/j.1475-4754.2003.00133.x.

[3] R.J.H. Wanhill, "Case Histories of Ancient Silver Embrittlement", Journal of Failure Analysis and Prevention, 11 (2011) 178-185, DOI: 10.1007/ s11668-010-9429-5.

[4] J.P. Franey, G.W. Kammlott, T.E. Graedel, "The corrosion of silver by atmospheric sulfurous gases", Corrosion Science, 25 (1985) 133-143, DOI: 10.1016/0010-938X(85)90104-0.

[5] V. Costa, "The deterioration of silver alloys and some aspects of their conservation", Reviews in Con- servation, 2 (2001) 18-34, DOI: 10.1179/sic.2001.46. Supplement-1.18.

[6] G. Basilissi, A. Cagnini, "Ricerca e sperimentazione di nuove metodologie di pulitura dell'argento e messa a punto di idonei formulate protettivi", in: Proc. Lo stato dell'arte 14, L'Aquila, Italy, October 20-22, 2016, Nardini Editore, Firenze 2016, pp. 99-107.

[7] T. Palomar, B. Ramírez Barat, E. García, E. Cano, "A comparative study of cleaning methods for tarnished silver", Journal of Cultural Heritage, 17 (2016) 20-26, DOI: 10.1016/j.culher.2015.07.012.

[8] T. Palomar, M. Oujja, I. Llorente, B. Ramirez Barat, M.V. Canamares, E. Cano, M. Castillejo, "Evaluation of laser cleaning for the restoration of tarnished silver artifacts", Applied Surface Science, 387 (2016) 118-127, DOI: 10.1016/j.apsusc.2016.06.017.

[9] R. Fontana, M.C. Gambino, M. Greco, L. Marras, M. Materazzi, E. Pampaloni, L. Pezzati, "High-precision surface analysis of the roughness of Michelangelo's David", Proc. SPIE 5146 (2003) 236-243, DOI: 10.1117/12.501252.

[10] G. Y. Sirat, "Conoscopic holography. I. Basic principles and physical basis", Journal of the Optical Society of America A, 9 (1992) 70-83, DOI: 10.1364/ JOSAA.9.000070.

[11] P. Carcagni, C. Daffara, R. Fontana, M.C. Gambino, M. Mastroianni, C. Mazzotta, E. Pampaloni, L. Pezzati, "Optical micro-profilometry for archaeology”, Proc. SPIE 5857 (2005) 58570F, DOI: 10.1117/12.612527.

[12] C. Colombo, C. Daffara, R. Fontana, M.C. Gambino, M. Mastroianni, E. Pampaloni, M. Realini, A. Sansonetti, "Evaluation by Laser Micro-Profilometry of Morphological Changes Induced on Stone Materials by Laser Cleaning", in: Laser in the Conservation of Artworks (Proceedings of LACONA VI Conference), Vienna, Austria, September 21-25, 2005, M. J. Nimmrichter, W. Kautek, M. Schreiner (Eds.), Springer proceedings in physics 116, Springer, Berlin 2007, pp. 523-526, DOI: 10.1007/978-3-540-72310-7_62.

[13] R. Fontana, A. Dal Fovo, J. Striova, L. Pezzati, E. Pampaloni, M. Raffaelli, M. Barucci, "Application of non-invasive optical monitoring methodologies to follow and record painting cleaning processes", Ap- 
plied Physics A, 121 (2015), 957-966, DOI: 10.1007/ s00339-015-9505-5.

[14] Bharat Bhushan, "Surface Roughness Analysis and Measurement Techniques", in: Modern Tribology Handbook, CRC Press, Boca Raton, London, New York, Washington D.C. 2000, pp. 49-119, DOI: $10.1201 / 9780849377877$.

[15] N. Gaburro, G. Marchioro, C. Daffara, "A versatile optical profilometer based on conoscopic holography sensors for acquisition of specular and diffusive surfaces in artworks", Proc. SPIE 10331 (2017) 103310A, DOI: 10.1117/12.2270307.

[16] D.J. Whitehouse, Handbook of Surface Metrology, Institute of Physics Publishing, Bristol U.K. 1994.

[17] R. Leach (Ed), Characterisation of Areal Surface Texture, Springer, Heidelberg 2013.
[18] ISO 25178-2, Geometrical Product Specifications (GPS) - Surface texture: Areal - Part 2): Terms, Definitions and Surface Texture Parameters, International Organization for Standardization 2012.

[19] J.J. Coronado, "Effect of Abrasive Size on Wear" in: M. Adamiak (Ed.), Abrasion Resistance of Materials, InTech, 2012, pp. 167-184.

[20] K. Kato, K. Adachi, "Wear Mechanism" in: Bharat Bhushan (Ed.), Modern Tribology Handbook, CRC Press, Boca Raton, London, New York, Washington D.C. 2000, pp. 273-300, DOI: 10.1201/9780849377877.

[21] M. Bigerelle, T. Mathia, S. Bouvier, "The multiscale roughness analysis and modelling of abrasion with the grit size effect on ground surface", Wear, 286-287 (2012) 124-135, DOI: 10.1016/j. wear.2011.08.006. 